\title{
Array Tomography and Beam Deceleration - High-Throughput Imaging with the ZEISS GeminiSEM using Atlas 5 and Beam Deceleration
}

\author{
Angert Isabel ${ }^{1}$, Kirmse Robert $^{1}$, Marlene Thaler $^{1}$ and Kirk Czymmek ${ }^{1}$ \\ 1. Carl Zeiss Microscopy GmbH, Jena Germany
}

Utilizing scanning electron microscopy (SEM) to investigate the 3D morphology of cells and tissue has become more and more important in the in the field of biological and medical research. In general two major methods can be distinguished serial block face imaging and Array Tomography [1]. In serial blockface imaging the whole block of resin embedded biological tissue is introduced into the SEM chamber. Then a microtome [2] or focused ion beams [3] are used to cut off a thin top layer of a few $\mathrm{nm}$. An SEM image of the fresh blockface is acquired and the process repeated. Conversely Array Tomography starts by collecting conventional serial section of the resin embedded sample either manually or automatically on larger solid supports which are then introduced into the SEM. Automated image acquisition software is then utilized to record images of hundreds of serial section. Both methods generate hundreds to thousands of sequential images that can be re-aligned to form the 3D volume of the initially sectioned sample.

Recording quality SEM images especially of resin embedded biological samples depends on a variety of parameters. However assuming a well prepared sample that can be investigated without charging artefact, the main question remains: "How much can the pixel dwell time be reduced while still obtaining the optimal signal to noise required?" In other words, besides resolution highest speed and optimized contrast in the images are the key factors for high throughput data acquisition. Here we investigate the influence and benefits of beam deceleration at the sample stage ("Tandem Deceleration"), on high throughput imaging. We can show that "Tandem Deceleration" allows retaining the same signal to noise while decreasing the acquisition time significantly.

An additional potential between the pole piece of the SEM and the sample stage has several effects. First electrons with a primary energy dependent on the electronic high tension (EHT) are decelerated on their way to the sample by the bias voltage and as a result hit the sample with a landing energy reduced by the potential applied to the sample stage. Electrons leaving the sample on their way back to the detector are accelerated by the same bias voltage towards the detectors.

This electric potential leads directly to a higher signal on the inlens SE detector which allows to reduce the scan speed by a factor of four while retaining the same signal to noise ratio in the image (see Fig. $1 \& 2)$. For surface investigations of resin embedded samples diode type back scatter detectors (BSD) and low electron energies are preferred to avoid charging and beam damage. However the efficiency of BSD detectors for electrons below $2 \mathrm{keV}$ is generally reduced. Yet an additional bias voltage (e.g. $5 \mathrm{kV}$ ) introduced a remarkable gain in contrast even at landing energies of $1.5 \mathrm{keV}$ (See Fig. 1\&2). Moreover the pixel dwell times can again be reduced to benefit from the additional speed gain.

Utilizing the newly implemented "Tandem Deceleration" in the ZEISS GeminiSEM we could show an at least up to four fold faster acquisition time for single image frames alongside a boost in detection efficiency. In combination with the ZEISS Atlas 5 software package this provide an easy access to large area and hierarchical imaging at higher speeds previously not possible. 


\section{References:}

[1] Micheva KD, Smith SJ. Neuron. 2007 Jul 5;55(1):25-36. Erratum in: Neuron. 2007 Sep 6;55(5):824. [2] Denk W, Horstmann H. PLoS Biol. 2004 Nov; 2(11):e329. Epub 2004 Oct 19. [3] Narayan K et al, Subramaniam S5, Struct Biol. 2014 Mar;185(3):278-84. Epub 2013 Dec 1.
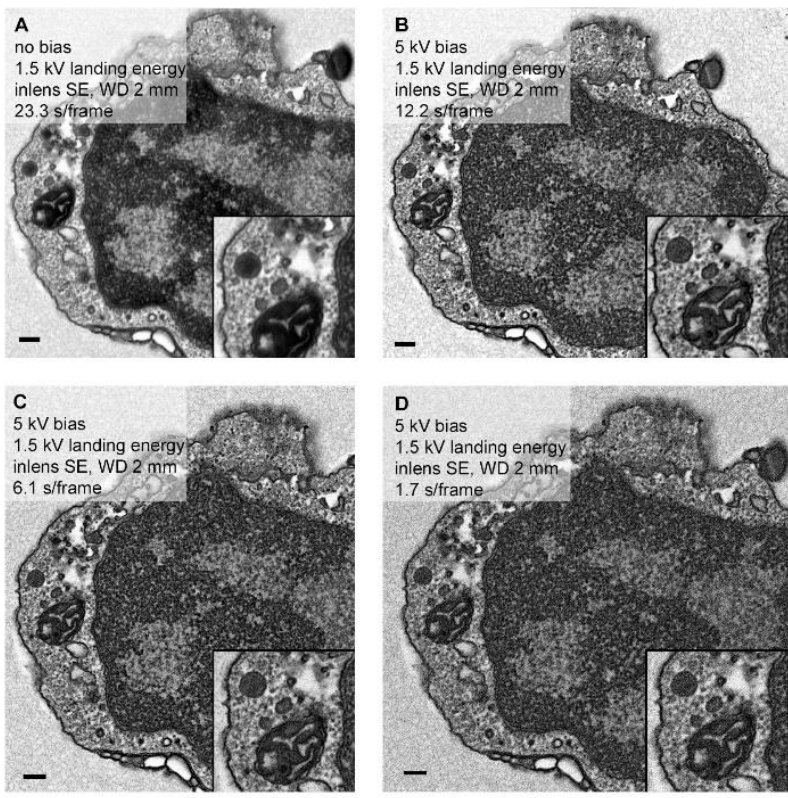

Figure 1. Inlens SE detector with and without beam deceleration. A-D, all images are recorded with $3072 \times 2304$ pixels. The acquisition time and bias on/off is stated on the images. B-D The image series demonstrates that acquisition time can be reduced significantly while retaining $(\mathbf{C}, \mathbf{D})$ or even improving image quality (B) over (A).
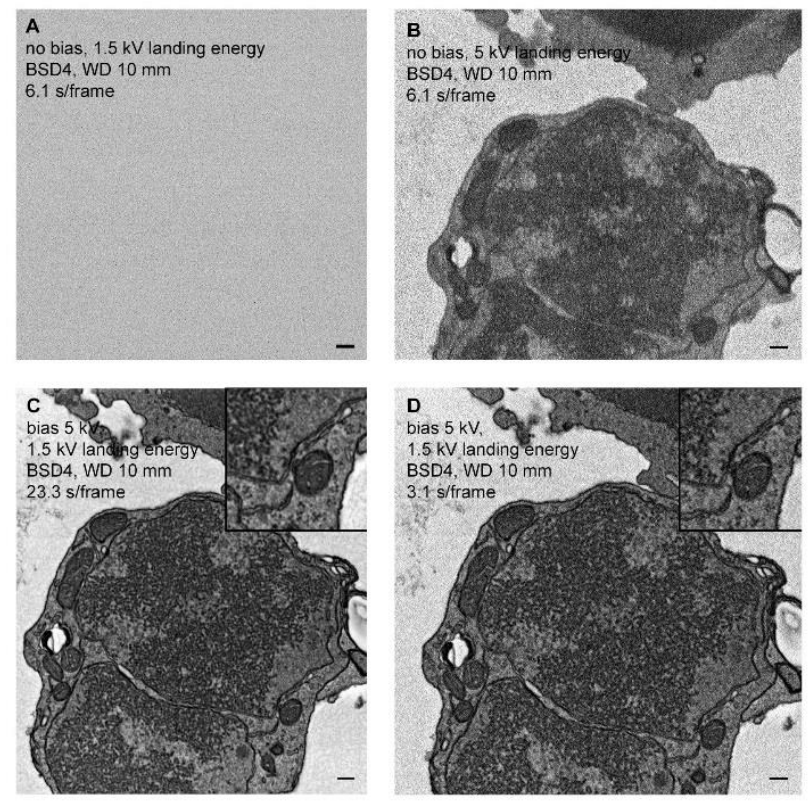

Figure 2. Post column backscatter detector (BSD) with and without beam deceleration. A-D, all images are recorded with $3072 \times 2304$ pixels. The acquisition time and bias on/off is stated on the images.

Here a working distance (WD) of $10 \mathrm{~mm}$ was chosen to accommodate the altered trajectory of the electrons in the bias field. A-B. Under these conditions low $\mathrm{kV}$ BSD images are not possible, only at higher $\mathrm{kV}$ (here $5 \mathrm{kV}$ ) can the sample be detected with the fast frame rates use. (CD) Using Tandem Deceleration however we can use low landing energies to obtain high-quality images $(\mathbf{C})$, but are also able to use high frame rates while retaining signal to noise (D).

Serial sections of immune cells from zebrafish medulla were placed directly on a silicon wafer (Sample was kindly provided by Irene Wacker et al. Bioquant, University Heidelberg Germany). Scale bars $200 \mathrm{~nm}$ 